\title{
Instrumento para a atenção integral: do pré-natal ao puerpério
}

\section{Instrument for an integrated: pre-natal and post-natal care}

\section{Resumo}

Alexandre Borges Fortes ${ }^{1}$ Maria Inez Padula Anderson ${ }^{2}$ Miriam Schenker ${ }^{3}$

Este trabalho apresenta um instrumento elaborado para auxiliar o médico de Família e Comunidade, e também profissionais da saúde, no aprendizado e na prática de uma abordagem integral do pré-natal ao puerpério. Acredita-se que, abordando não apenas a gestante, mas também o casal (e por vezes a família), cuidando do bem-estar biopsicossocial, seja possível gerar resiliência e, dessa forma, com base nos estudos em psiconeuroendocrinoimunologia, promover a saúde e diminuir as chances de adoecimento e/ou complicações como parto prematuro, pré-eclampsia, infecções, dentre outras.

\begin{abstract}
This work presents an instrument to aid the general practitioner and other health professionals in providing integrated healthcare during the pre-and post-natal period. It is believed that through an integrated approach to the psychosocial well-being not only of the pregnant women but of the couple (and at times of the whole family) it is possible to create resilience and with this, according to pshyconeuroendocrinoimmunology studies, promote health and lower the chances of falling ill and/ or having complications (preterm delivery, preeclampsia, infections among others).
\end{abstract}

Palavras-chave: Cuidado Pré-Natal; Assistência Integral à Saúde; Promoção da Saúde; Saúde da Família.

Key Words: Prenatal care; Comprehensive

Health Care; Health Promotion; Family Health.

\footnotetext{
${ }^{1}$ Médico, Residência em Medicina de Família e Comunidade, Hospital Universitário Pedro Ernesto, Universidade do Estado Rio de Janeiro, Rio de Janeiro, Brasil.

${ }^{2}$ Médica, Professora de Medicina de Família e Comunidade, Departamento de Medicina de Família e Comunidade, Faculdade de Ciências Médicas, Universidade do Estado Rio de Janeiro, Rio de Janeiro, Brasil.

${ }^{3}$ Psicóloga, Doutora em Ciências Instituto Fernandes Figueira, Fundação Oswaldo Cruz, Departamento de Medicina de Família e Comunidade, Faculdade de Ciências Médicas, Universidade do Estado Rio de Janeiro, Rio de Janeiro, Brasil.
} 


\section{Introdução}

Os estudos de psiconeuroendocrinoimunologia nos revelam mecanismos pelos quais fatores estressantes podem predispor o indivíduo a adoecer, e também que a promoção da resiliência tende a incrementar a saúde, aumentando, especialmente, a capacidade de lidar com os diferentes tipos de estresse ${ }^{1-13}$.

Os ciclos de vida são períodos potencialmente conturbados na vida das famílias; são momentos de mudanças em sua dinâmica, estrutura e seu funcionamento. Alguns podem ser difíceis, como, por exemplo, o falecimento de um ente querido, o nascimento do primeiro filho e a saída de casa dos filhos; outros nem tanto.

Os conhecimentos adquiridos por meio dos estudos dos ciclos de vida familiar ${ }^{14-19}$ associados aos de psiconeuroendocrinoimunologia levaram os autores a criar um instrumento capaz de facilitar o aprendizado e a prática de uma abordagem integral à gestante e ao casal no ciclo de vida que se inicia no pré-natal estendendo-se até o puerpério.

A avaliação dos ciclos de vida pode ser vista como uma oportunidade de preparo do indivíduo e da família para lidar com essas etapas, instrumentalizando-os para desenvolverem atitudes e comportamentos resilientes, diminuindo, dessa forma, o nível de estresse e promovendo saúde.

A psicoprofilaxia tem a finalidade de orientar a gestante nos seus aspectos físicos e emocionais, preparandoa para uma gravidez mais saudável e para uma visão positiva no momento do parto ${ }^{20}$, e de estimulá-la, por meio da reflexão, para aspectos relevantes da forma como ela e o pai poderão cuidar do bebê, em uma perspectiva biopsicológica. Pode ser iniciada quando o casal manifesta o desejo de ter um filho ou na primeira consulta do pré-natal, estendendo-se até o terceiro ano de vida da criança.

O presente instrumento associa à consulta médica tradicional meios de abordar e preparar a gestante e o casal, não somente para o parto, mas para a vida que se segue.

\section{Objetivos}

O objetivo deste trabalho é apresentar um ins- trumento que visa facilitar, ao médico de Família e Comunidade (MFC) em particular e aos profissionais de saúde em geral, uma abordagem integral no pré-natal associada à idéia dos ciclos de vida, aplicada à saúde da família.

\section{Material e Método}

Este instrumento foi elaborado com base em revisão da literatura ${ }^{21-29}$, aulas teóricas e estudo em grupo a partir de dramatizações simulando consultas do MFC através do ciclo de vida familiar, durante o curso intitulado Abordagem Familiar para residentes em Medicina de Família e Comunidade do Departamento de Medicina Integral Familiar e Comunitária (DMIF) da Universidade do Estado do Rio de Janeiro (UERJ).

Os dados do instrumento referentes à psicoprofilaxia foram formatados tendo como ponto de partida a descrição das etapas do ciclo de vida familiar de Wagner e colaboradores $^{18}$, conjugada às dramatizações devidamente registradas e posteriormente analisadas pelos participantes do curso. O artigo foi escolhido, a partir da revisão de literatura, por abordar didaticamente as diferentes etapas do ciclo vital familiar, incluindo tópicos de prevenção a serem pensados pelo médico ${ }^{18}$.

A cada semana, uma etapa do ciclo era dramatizada pelos residentes, de tal forma que uns representavam o papel do médico, outros do paciente/da família com determinado problema - podendo ser desde uma dificuldade social, familiar, orgânica e/ou emocional - e o restante dos residentes observavam e faziam anotações sobre os pontos positivos e negativos daquilo a que assistiam, e dos aspectos que thes pareciam relevantes para serem abordados nestas situações. Em seguida, as anotações eram discutidas em grupo e o produto desse trabalho, organizado em uma tabela.

O instrumento apresentado contempla uma das etapas do ciclo de vida da família organizadas nessa tabela: do pré-natal ao puerpério.

A lista de tópicos que deve ser abordada nas consultas pode variar de acordo com as características de cada população, tornando o instrumento flexível e aplicável 
a distintas realidades socioantropológicas e culturais. Por exemplo: em uma comunidade que vive diariamente violência e falta de saneamento básico, os temas propostos provavelmente serão diferentes dos de outra, cujo meio é livre desses problemas.

Assim, cabe ao MFC melhor adequar quais temas desta lista melhor se aplicarão à realidade de sua comunidade, assim como criar novos quesitos de acordo com o diagnóstico comunitário.

No instrumento apresentado estão dispostos itens que os autores julgaram pertinentes à realidade da população atendida pelos residentes de Medicina de Família e Comunidade: moradores dos subúrbios da cidade do Rio de Janeiro próximos do Hospital Universitário Pedro Ernesto (HUPE).

\section{Resultados}

O instrumento elaborado consta de uma tabela separada por três colunas de acordo com os primeiros três trimestres de vida do bebê - do diagnóstico da gravidez até a $12^{\mathrm{a}}$ semana; da $13^{\mathrm{a}}$ à $24^{\mathrm{a}}$ semanas, e da $25^{\mathrm{a}}$ até o parto - e duas categorias de cuidados; uma acima e outra abaixo.

A primeira parte, orientada pela linha na cor amarela, detalha os exames e condutas a serem executadas pelo médico: o exame obstétrico inclui avaliação do bem-estar materno-fetal com medidas de fundo de útero, IMC, PA, ausculta dos BCF e outros. Faz parte também desta etapa avaliar e resolver intercorrências que, porventura, surjam, como, por exemplo: febre, corrimento vaginal, disúria, sangramentos e outros. Certos exames são recomendados pelo Ministério da Saúde para todas as gestantes; por esse motivo são mencionados como exames de rotina.

A segunda parte, na cor verde, refere-se à psicoprofilaxia, na qual são abordados temas como: o trabalho com os medos e tabus que causam insegurança e aflição, do período gestacional aos primeiros três anos da criança; o fortalecimento da auto-estima do casal e da gestante; a conscientização do casal sobre as mudanças na dinâmica familiar e no ciclo de vida que se passa - que tipo de vivência cada um teve na sua família de origem e o que desejam passar para seus filhos -; o fortalecimento das atitudes e dos comportamentos resilientes; a facilitação, promoção da integração do casal; a promoção da participação do pai em todos os processos; o desenvolvimento de vínculo mãepai-filho; a preparação para maternagem e paternagem; o empoderamento da gestante, tornando-a ativa; a prevenção da depressão pós-parto e psicose puerperal.

A terceira parte (correspondente ao verso da tabela) refere-se à primeira consulta da gestante no pré-natal, na qual são abordadas questões básicas como anamnese clínica, obstétrica, ginecológica e sexual; exame físico completo; solicitação dos exames de rotina e avaliação de riscos mais urgentes, como, por exemplo, o risco de violência contra a mulher, o uso de drogas, álcool, vacinação, dentre outros.

A tabela deverá ser utilizada no momento da consulta de acordo com o estágio da gestação. Sugere ao médico e ao profissional de saúde temas e aspectos a serem observados que propiciam a abordagem de questões relevantes para o acompanhamento e a psicoprofilaxia, ajudando na reflexão conjunta e na proposição de resolução de problemas.

Espera-se que o médico possa utilizar este instrumento como ferramenta de consulta rápida durante seus atendimentos em pré-natal, do mesmo modo como usaria ocasionalmente um calendário. Cada quesito de psicoprofilaxia proposto pode ser motivo de longas dissertações. Portanto, não basta apenas ler os itens da tabela que compõem o instrumento. É importante que o médico estude detalhadamente cada um deles para a sua boa utilização.

\section{Discussão}

$\mathrm{Na}$ revisão da literatura, encontramos alguns textos e livros que abordam o Ciclo de Vida familiar. Nosso objetivo era o de angariar material no qual o Ciclo de Vida fosse utilizado como uma ferramenta para a abordagem da família pelo médico. O livro editado por Carter e MacGoldrick ${ }^{15}$ descreve o adoecimento da família e suas mudanças ao longo deste Ciclo; o livro de Eizirik, Kapczinski e Bassols ${ }^{16}$ segue a mesma linha do anterior sob a ótica psicanalítica; o capítulo de Fernandes e Curra ${ }^{17}$ intitulado Ferramentas de abordagem 


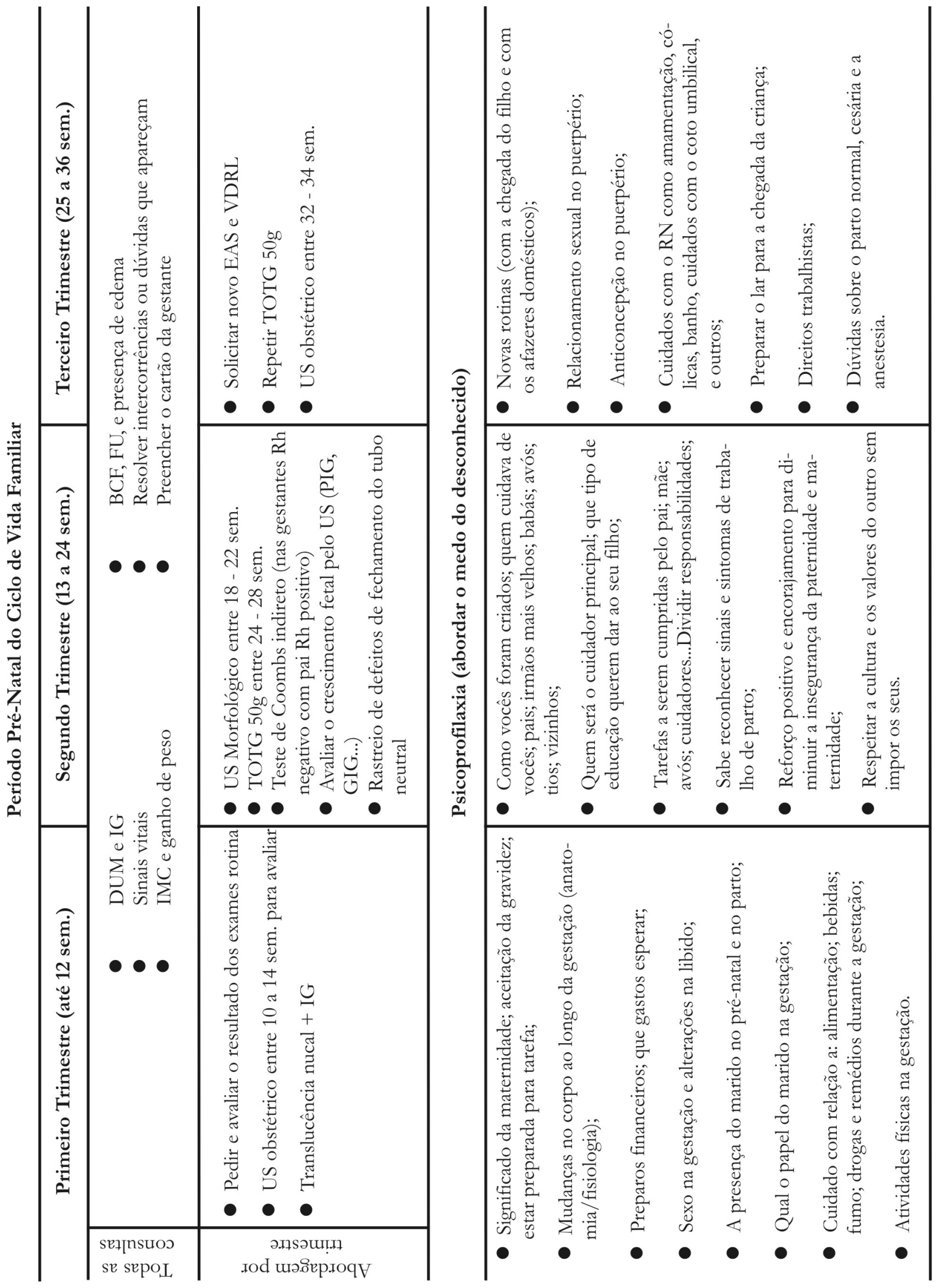




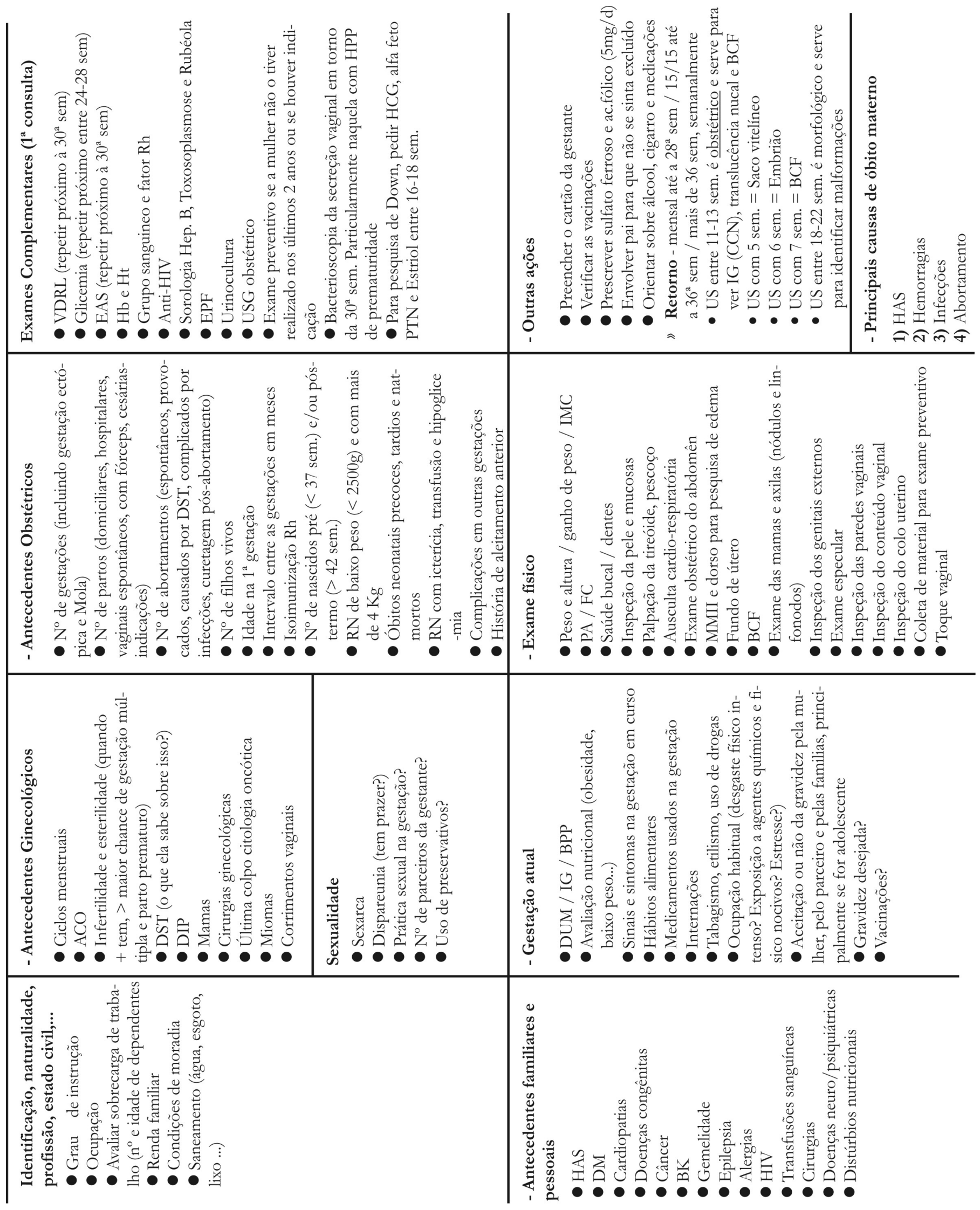


da família traz contribuições acerca dos diferentes estágios do ciclo de vida e as tarefas que deverão ser cumpridas pela família em cada um deles. Bernal e colaboradores ${ }^{14}$ classificam as etapas do ciclo de vida segundo seu movimento - de formação, extensão, contração e dissolução - e não se preocupam com o detalhamento dos vários estágios do ciclo de vida das famílias; o livro Trabalhando com Famílias ${ }^{19}$ apresenta um capítulo acerca da abordagem do ciclo vital familiar pelo médico de forma bem sucinta. Já o artigo de Wagner e colaboradores ${ }^{18}$ contribui com especificações de diversas etapas do ciclo de vida familiar, relacionando-as com a abordagem integral do médico.

A ampliação do espectro das etapas do ciclo de vida da família, que teve como ponto de partida o artigo supracitado de Wagner e colaboradores, aliada às dramatizações e discussões reflexivas, possibilitou a criação de um instrumento minucioso e detalhado para o atendimento de qualidade, tanto às grávidas quanto ao casal que vive a etapa do ciclo vital do pré-natal ao puerpério.

Outrossim, o instrumento foi elaborado visando facilitar o aprendizado e a prática de uma abordagem integral da mulher e da família na Atenção Primária no período per e peri-gestacional. Acredita-se que, com ele em mãos, o médico possa ter uma forma de recordar determinados itens importantes de serem abordados durante uma consulta. Espera-se que, com o tempo, o instrumento possa ser cada vez mais utilizado pelos profissionais de saúde, incluindo-se aí o MFC.

Cabe ressaltar que o instrumento sistematizado ainda não foi utilizado com a freqüência que permita saber se o seu uso poderá de fato melhorar ou facilitar o aprendizado da abordagem integral.

\section{Conclusão}

Estímulos externos capazes de piorar a qualidade de vida e gerar sofrimento sempre estarão presentes, e não há nada que se possa fazer a respeito disso. Mas é possível aumentar nossa resistência ao estresse, nossa resiliência. Dessa forma, pretende-se contribuir para modular de maneira autônoma esta resposta de tal modo que se possam diminuir reações emocionais negativas com repercussões sistêmicas. Esse é o maior objetivo da psicoprofilaxia trabalhada ao longo do ciclo de vida da pessoa e da família pelo médico de família.

O instrumento aponta para a pertinência e a necessidade de se incluir o marido/companheiro/namorado nas consultas do pré-natal ao puerpério. O casal será responsável pela criança, ora no ventre. Estimular reflexões acerca de um relacionamento saudável dos futuros pais poderá garantir o bem-estar do bebê e de sua família.

O treinamento teórico-prático específico em relação à abordagem integral no pré-natal é fundamental não somente pela importância do assunto, mas também pela sua maior complexidade, conforme se depreende das reflexões e análises ao longo do artigo. Acreditamos que, com o auxílio do instrumento criado, possa-se facilitar o aprendizado e a prática do médico de família e comunidade acerca da abordagem da integralidade do pré-natal ao puerpério e, assim, auxiliar na promoção da saúde das famílias que se encontram nessa etapa do ciclo vital.

\section{Referências}

1. Anisman H, Baines M, Berczi I, Bernstein C, Blennerhassett M, Gorczynski R, et al. Neuroimmune mechanisms in health and disease: 2. Disease. Can Med Assoc J. 1996; 155(8): 10751082.

2. Ballieux RE. Impact of mental stress on the immune response. J Clin Periodontol. 1991; 18 (6): 427-430

3. Biondi M, Zannino L. Psychological stress, neuroimmunomodulation, and susceptibility to infectious diseases in animals and man: a review. Psychother Psychosom. 1997; 66(1):3-26.

4. Cohen S, Tyrrell D, Smith A. Negative life events, perceived stress, negative affect, and susceptibility to the common cold. J Pers Soc Psychol. 1993; 64: 131-140.

5. Coussons-Read M, Okun M, Schmitt M, Giese S. Prenatal stress alters cytokine levels in a manner that may endanger human pregnancy. Psychosom Med. 2005; 67: 625-631. 6. Dole N, Savitz D, Hertz-Picciotto I, Siega-Riz A, McMahon M, Buckens P. Maternal stress and preterm birth. 
Obstet Gynecol Surv. 2003; 58: 365-366.

7. Gennaro S, Fehder WP. Stress, immune function, and relationship to pregnancy outcome. Nurs Clin North Am. 1996; 31: 293-302.

8. Kaschka W. How are psychological processes, the neuroendocrine system and immune system integrated? Z Klin Psychol Psychopathol Psychother. 1996; 44(3): 280-289.

9. Kiecolt-Glaser J, Glaser R. Psychoneuroimmunology: can psychological interventions modulate immunity? J Consult Clin Psychol. 1992; 60(4): 569-575

10. Kropiunigg U. Basics in psychoneuroimmunology. Ann Med. 1993; 25(5): 473-479.

11. Leonard B, Miller K. Stress, the immune system and psychiatry. Ireland: J.Wiley \& Sons; 1995. 238 p.

12. Livingston IL, Otado JA, Warren C. Stress, adverse pregnancy outcomes and African-American females. J Natl Med Assoc. 2003; 95: 1103-1109.

13. Orr S, James S, Casper R. Psychosocial stressors and low birthweight: development of a questionnaire. J Dev Behav Pediatr. 1992;13: 343-347.

14. Bernal IL e colaboradores. Manual para la intervención em la salud familiar. Cuba: Ministerio de La Salud Pública da República de Cuba. Grupo assessor metodológico de estúdios de salud de la familia: 2001.

15. Carter B, McGoldrick M. As mudanças no ciclo de vida familiar. Porto Alegre: Artmed; 2001. 510 p.

16. Eizirik CL, Kapczinski F, Bassols AMS. O ciclo da vida humana: uma perspectiva piscodinâmica. Porto Alegre: Artmed; 2001. 200 p.

17. Fernandes CLC, Curra LCD. Ferramentas de abordagem da família. In SBMFC (org.) PROMEF. Porto Alegre: Artmed, Panamericana; 2006. p.11-42.

18. Wagner HL, Talbot Y, Wagner ABP, Oliveira E. Ferramenta de avaliação para situações indefinidas e manobras preventivas em saúde da família - ciclo de vida das famílias. Rev. Méd. Paraná. 1999: 57(1/2): 22-27, tab. 19. Wilson L, Bader E. Ciclo de vida das famílias. In: Wilson L (ed.). Trabalhando com famílias. Livro de trabalho para residentes. Tradução de Wagner HL. Curitiba: SMS-Curitiba, 1996, p.30-31.

20. Valloto S, Almeida D, Caparroz S. Psicoprofilaxia da gravidez: o preparo físico e emocional para a gestantes. Revista Arquivos do Mudi. 2006; (10) (supl1):269-270.

21. Brasil. Ministério da Saúde. Secretaria de Atenção à Saúde, Departamento de Ações Programáticas Estratégicas. Pré-natal e puerpério: atenção qualificada e humanizada. Manual Técnico, Brasília (DF): Ministério da Saúde; 2005.160 p.

22. Dahlke R, Dahlke M, Zahn V. O caminho para a vida: gravidez e parto levando em conta o ser humano como um todo. São Paulo: Cultrix; 2005.

23. Duncan M, Giugliani E, Duncan B, Gaio D. Assistência pré-natal e puerpério: medicina ambulatorial: condutas de atenção primária baseadas em evidências. Porto Alegre: Artmed; 2004. 1600 p.

24. FEBRASGO. Comissão Nacional Especializada de Assistência Pré-Natal. Assistência Pré-Natal: manual deorientação; disponível em: <http://www.gosites.com.br/ sggo/pdfasp?path=302553jh\%7Cggeyny7zmls2rjl4\& arq $=\mathrm{rcq} \% 7 \mathrm{Chp} 6788 \% 3 \mathrm{~A} 2 \mathrm{vml}$. Acesso em 18 de outubro de 2007.

25. Ferrari A. Acompanhamento Pré-Natal. PROMEF. Ciclo 1 - Módulo 4. Porto Alegre: Artmed, Panamericana; 2006. $176 \mathrm{p}$.

26. Garibaldi R. Como funciona um bebê? Rio de Janeiro: Rúbio; 2003. 87 p.

27. Melillo A, Ojeda E. Resiliência: descobrindo as próprias fortalezas. Porto Alegre: Artmed; 2005. 160 p.

28. Vasques F. Pré-natal - um enfoque multiprofissional. Rio de Janeiro: Rúbio; 2006. 118 p.

29. Zugaib M, Ruocco R. Pré-natal. São Paulo: Atheneu; 2005. $396 \mathrm{p}$. 


\section{Endereços para correspondências:}

Hospital Universitário Pedro Ernesto

Dpto. de Medicina Familiar e Comunitária

Av. Vinte e Oito de Setembro $\mathrm{n}^{\circ} 77$

Vila Isabel - Rio de Janeiro RJ

CEP: 20551-030

\section{Endereço eletrônico:}

admint@uerj.br 